\title{
Maize-like ionic liquid@polyaniline nanocomposites for high performance supercapacitor
}

https://doi.org/10.1515/epoly-2019-0032

Received October 24, 2018; accepted January 07, 2019.

\begin{abstract}
In this study, ionic liquids (IL) containing carboxyl and different alkyl chains were fabricated and used to dope polyaniline (PANI). The results revealed that IL@PANI composites could be facilely obtained via template-free polymerization of aniline using ammonium persulfate as the oxidant. The as-prepared IL@PANI composites were measured by FT-IR, XPS, and SEM. Electrochemical performances of IL@PANI nanocomposites were investigated by cyclic voltammetry and galvanostatic charge/discharge. The results indicate that the alkyl chains of ILs have an important influence on the morphology and capacitance performance of IL@PANI electrode materials. With the shorter alkyl group in ILs, IL@PANI materials presented higher specific capacitance. Especially, 1-vinyl-3-carboxymethyl-imidazolium chloride ([VCMIm]Cl)@PANI composite presented the highest specific capacitance. Cycling performance measurement demonstrated that $82 \%$ capacitance retention could be achieved after 1000 cycles in $0.5 \mathrm{M} \mathrm{H}_{2} \mathrm{SO}_{4}$ aqueous solution. Therefore, our strategy provides a new technique for PANI nanocomposites with tunable morphology and high performance.
\end{abstract}

Keywords: ionic liquid; nanorod; polyaniline; supercapacitor

\footnotetext{
* Corresponding author: Yubing Xiong, Key Laboratory of EcoEnvironment-Related Polymer Materials, Ministry of Education; College of Chemistry and Chemical Engineering, Northwest Normal University, Lanzhou 730070, China; Department of Chemistry, College of Science, Zhejiang Sci-Tech University, Hangzhou 310018, P. R. China, e-mail: yubing_xiong@163.com. Honghong Song, Jing Zhang and Pengfei Song, Key Laboratory of Eco-Environment-Related Polymer Materials, Ministry of Education; College of Chemistry and Chemical Engineering, Northwest Normal University, Lanzhou 730070, China.
}

\section{Introduction}

In view of the increasing demands for sustainable energy materials, supercapacitors (also defined as electric double-layer capacitors and ultracapacitors) have been one of the most popular topics in the many fields, such as consumer electronics, portable energy sources, high power actuators, and hybrid electric vehicles, etc (1-5). Previous studies have demonstrated that electrode materials play a crucial role in supercapacitors (6). Therefore, great efforts have been devoted to explore novel electrode materials. Carbon materials possess many excellent advantages, including high specific surface area, good thermal and chemical stability, low elastic modulus, etc (7-9). As a result, carbon materials such as carbon nanotube, graphene, and porous carbon have been one of the moststudied electrode materials (10-13). On the other hand, carbon materials also suffer from the disadvantages of low specific capacitance and low preparation efficiency (14). On the contrary, transition metal oxides and conducting polymers are being regarded as promising and alternative electrode materials, because they can offer much higher specific capacitance and energy density than carbon materials $(15,16)$. Nonetheless, the high cost has greatly limited their practical applications (17).

Compared with the expensive metal oxides, conducting polymers are becoming more and more attractive because they can afford higher charge density with low cost (18). Till now, the most widely studied conductive polymers for supercapacitor electrode materials are polyaniline (PANI), polypyrrole (PPy), polythiophene (PT) and their derivatives (19). Especially, PANI has attracted extensive interest owing to its good environmental/thermo stability, high conductivity, and easy modification by mediating the oxidation state (20-23). Therefore, PANI has been one of the most promising conducting polymers and widely applied in chemical sensors, energy conversion and storage, microelectronics, etc. Recently, PANI has been developed as a promising candidate for the electrode material of 
supercapacitor $(24,25)$. The studies revealed that the microstructure has a critical effect on the capacitance performance of PANI (26-28). Doping PANI with different acids is an efficient technique to regulate its morphology and improve the capacitance performance. Compared with inorganic acid-based dopants, PANI doped by organic acid can offer better stability and compatibility (29).

During the past decades, ionic liquids (ILs) have grown to be an attractive "green" media and eminent material because of their non-flammable, low vapor pressure, good chemical and thermal stability, high conductivity, designable structure and polarity, and broad electrochemical window (30-33). In the light of their higher decomposition temperature and excellent chemical stability, ILs have been explored as promising electrolytes for electrochemistry and supercapacitors (34-37). T. Attri et al. investigated the interactions between ILs of ammonium and imidazolium families and PANI. And the strength of hydrogen bonding between ILs and PANI from semiempirical calculations was also determined. Their results demonstrated that PANI-IL additives could be used as electrolytes in dye-sensitized solar cells (38). M. Zigon et al. reported that nanostructured PANI with various morphologies could be obtained in acidic aqueous medium containing ILs with imidazolium, pyridinium and quaternary ammonium cations. It was found that the mole ratio of aniline/IL had great influence on the morphology and properties of formed PANIs (39). However, the physical and chemical properties of ILsstrongly rely on the structures of cation and anion (40). Therefore, it is still highly desired to improve the performance of PANI composites through tuning the structure of ILs (41). Herein, imidazolium-based ILs with carboxyl and different alkyl groups, which have different interactions with PANI, were prepared. Then, PANI was doped by the as-prepared functional ILs via in situ chemical oxidation polymerization. Distinguished from the previous works on IL@PANI composites, our results indicated that IL@PANI nanocomposites have the maizelike morphology and can offer much higher capacitance performance than pristine PANI. Our studies thus provide a new strategy for the fabrication of PANI nanocomposites with high performance.

\section{Experimental}

\subsection{Materials}

Aniline (An) was distilled under reduced pressure before use. All the imidazole compounds, 1-vinyl imidazole (VIm, 98\%), 1-butyl imidazole (BIm, 98\%), 1-hexyl imidazole (HIm, 99\%), and 1-methyl imidazole (MIm, 99\%) were distilled under reduced pressure before use and kept in fridge. 1-Allyl-3-carboxymethylimidazolium chloride ([ACMIm] Cl, 95\%) was purchased from Kaite Scientific and Technology Ltd and used as received. Ammonium persulfate $\left(\left(\mathrm{NH}_{4}\right)_{2} \mathrm{~S}_{2} \mathrm{O}_{8}\right.$, APS), and chloroacetic acid (CAA) were A.R. grade and used without further treatment. All other chemicals were commercially available and used as received.

\subsection{Characterization}

Proton and carbon nuclear magnetic resonance spectroscopy ( ${ }^{1} \mathrm{H}$ and ${ }^{13} \mathrm{C}$ NMR) were measured on a Brucker AM $400 \mathrm{MHz}$ spectrometer at $25^{\circ} \mathrm{C}$. Fourier transform infrared (FT-IR) spectra were recorded on a DIGIL FTS3000 spectrophotometer using $\mathrm{KBr}$ tablets. Morphology of samples was observed by scanning electron microscopy (SEM, JSM 6700F, Japan). X-ray photoelectron spectra (XPS) were taken with an ESCALAB 210 instrument. The reported values of the binding energy are relative to $\mathrm{C} 1 \mathrm{~s}$ taken as $285.0 \mathrm{eV}$. The excitation source was the nonmonochromatic $\mathrm{K}_{\alpha}$ radiation of a magnesium anode $(1253.6 \mathrm{eV})$ and X-ray gun was operated at $300 \mathrm{~W}$ power.

Electrochemical measurements were conducted on a three-electrode system in $0.5 \mathrm{M} \mathrm{H}_{2} \mathrm{SO}_{4}$ solution at R.T. (CHI 660e, Chenhua, shanghai, China). A glassy carbon electrode with a diameter of $3 \mathrm{~mm}$ was applied as the working electrode. It was fabricated as follows: An active material ink was prepared using one part (by mass) of IL@PANIs and 11 parts of $0.5 \mathrm{wt} \%$ Nafion in ethanol. After sonicating thoroughly, $10 \mu \mathrm{L}$ of the ink was dispensed onto GCE and completely cover the current collector with a thin film of active material. The loading weight was $0.10 \mathrm{mg}$ on the working electrode. The counter electrodeand reference electrode were platinum electrode and saturated calomel electrode, respectively. Cyclic voltammetry (CV) of the composites were performed between -0.2 and $0.8 \mathrm{~V}$ at differentscan rates. Galvanostatic charge/discharge (GCD) behavior was evaluated at the current densities of $1.0,2.0,3.0,5.0$ and $10.0 \mathrm{~A} \mathrm{~g}^{-1}$ with cutoff voltage of $0-0.8 \mathrm{~V}$. Electrochemical impedance spectroscopy measurements were carried out in the frequency range of $100 \mathrm{MHz}$ to $0.01 \mathrm{~Hz}$ with $5 \mathrm{mV}$ amplitude at room temperature.

\subsection{Synthesis of 1-methyl-3-carboxymethyl- imidazolium chloride ([MCMIm]Cl)}

Imidazolium-based ILs with different substitutions were prepared according to the references $(42,43)$. A typical 
process for the synthesis of [MCMIm] Cl was as follows: MIm (0.82 g, $10 \mathrm{mmol})$ and CAA $(0.95 \mathrm{~g}, 10 \mathrm{mmol})$ were dissolved in dichloromethane $(50 \mathrm{~mL})$. Afterwards, the mixture was stirred at $55^{\circ} \mathrm{C}$ for $48 \mathrm{~h}$ under a nitrogen atmosphere. Following that, the reaction mixture was precipitated from diethyl ether $(100 \mathrm{~mL})$. The as-obtained crude product was recrystallized twice in diethyl ether. The product was dried on vacuum for $24 \mathrm{~h}$ at room temperature. [MCMIm] Cl was obtained as white solid. All other ILs were prepared through the similar procedure by using different imidazoles and alkyl chlorides. ${ }^{1} \mathrm{H}$ and ${ }^{13} \mathrm{C}$ NMR spectra were provided in the supporting information (Figures S1-S8).

[MCMIm]Cl: ${ }^{1} \mathrm{H}$ NMR ( $\left.\mathrm{D}_{2} \mathrm{O}, 400 \mathrm{MHz}, \delta \mathrm{ppm}\right): 8.57$ (s, 1H), 7.31(s, 2H), 4.69(s, 2H), 3.79(s, 3H). ${ }^{13} \mathrm{C}$ NMR ( $\mathrm{D}_{2} \mathrm{O}$, $100 \mathrm{MHz}, \mathrm{ppm}$ ): $172.00,136.63,123.31,123.13,51.89,35.43$. mp: $189-195^{\circ} \mathrm{C}$.

1-Vinyl-3-carboxymethyl-imidazolium chloride ([VCMIm]Cl), 1-butyl-3-carboxymethyl-imidazolium chloride ([BCMIm]Cl) and 1-hexyl-3-carboxymethylimidazolium chloride ([HCMIm]Cl) were prepared according to the same method.

[VCMIm]Cl: ${ }^{1} \mathrm{H}$ NMR ( $\left.\mathrm{D}_{2} \mathrm{O}, 400 \mathrm{MHz}, \delta \mathrm{ppm}\right): 8.88(\mathrm{~s}$, $1 \mathrm{H}), 7.61(\mathrm{~s}, 1 \mathrm{H}), 7.37(\mathrm{~s}, 1 \mathrm{H}), 7.00 \sim 6.94(\mathrm{dd}, 1 \mathrm{H}), 5.65 \sim 5.61(\mathrm{dd}$, 1H), 5.26 5.24(dd, $1 \mathrm{H}), 4.60(\mathrm{~s}, 2 \mathrm{H}) .{ }^{13} \mathrm{C} \mathrm{NMR}\left(\mathrm{D}_{2} \mathrm{O}, 100 \mathrm{MHz}\right.$, ppm): 173.11, 138.50, 131.00, 126.94, 122.14, 112.85, 53.70. mp: $175-185^{\circ} \mathrm{C}$.

[BCMIm]Cl: ${ }^{1} \mathrm{H}-\mathrm{NMR}\left(\mathrm{D}_{2} \mathrm{O}, 400 \mathrm{MHz}, \delta \mathrm{ppm}\right): 8.92(\mathrm{~s}$, $1 \mathrm{H}), 7.53(\mathrm{~s}, 1 \mathrm{H}), 7.57(\mathrm{~s}, 1 \mathrm{H}), 4.73(\mathrm{~s}, 2 \mathrm{H}), 4.21(\mathrm{t}, 2 \mathrm{H}), 1.85(\mathrm{~s}$, $2 \mathrm{H}), 1.36(\mathrm{~s}, 2 \mathrm{H}), 0.97(\mathrm{~s}, 3 \mathrm{H}) .{ }^{13} \mathrm{C}$ NMR ( $\left.\mathrm{D}_{2} \mathrm{O}, 100 \mathrm{MHz}, \mathrm{ppm}\right)$ : 172.14, 136.15, 123.40, 121.93, 51.89, 49.36, 31.17, 18.74, 12.62 . Liquid at r. t.

[HCMIm]Cl: ${ }^{1} \mathrm{H}-\mathrm{NMR}\left(\mathrm{D}_{2} \mathrm{O}, 400 \mathrm{MHz}, \delta \mathrm{ppm}\right): 8.65(\mathrm{~s}$, $1 \mathrm{H}), 7.34(\mathrm{~s}, 1 \mathrm{H}), 7.39(\mathrm{~s}, 1 \mathrm{H}), 4.70(\mathrm{~s}, 2 \mathrm{H}), 4.11(\mathrm{t}, 2 \mathrm{H}), 3.90(\mathrm{~s}$, $2 \mathrm{H}), 1.78(\mathrm{~d}, 2 \mathrm{H}), 1.20(\mathrm{~s}, 4 \mathrm{H}), 0.75(\mathrm{~d}, 3 \mathrm{H}) .{ }^{13} \mathrm{C} \mathrm{NMR}\left(\mathrm{D}_{2} \mathrm{O}, 100\right.$ $\mathrm{MHz}, \mathrm{ppm}): 172.40,136.11,123.37,121.92$, 51.84, 49.65, 30.25, 29.04, 24.92, 21.69, 13.15. Liquid at r. t.

\subsection{Preparation of IL@PANI composites}

The preparation process of IL@PANI composites was as follows (44): An (0.20 g, $2 \mathrm{mmol})$ and [VCMIm] Cl (0.38 g, $2 \mathrm{mmol}$ ) were dissolved in $10 \mathrm{~mL}$ deionized water under magnetic stirring for $30 \mathrm{~min}$ at R.T.. After then, the solution was cooled to $0-5^{\circ} \mathrm{C}$ using an ice bath, and APS $(0.45 \mathrm{~g}$, $2 \mathrm{mmol}$ ) in $5 \mathrm{~mL}$ deionized water was added dropwise into the solution above. Afterwards, the reaction was performed for another $12 \mathrm{~h}$ at $0-5^{\circ} \mathrm{C}$. Finally, the precipitate was collected by filtration and cleaned three times using deionized water and ethanol, respectively. The product was dried at $50^{\circ} \mathrm{C}$ for $12 \mathrm{~h}$, and was obtained as a dark green powder. All other
IL@PANI composites were prepared according to similar method except for changing ILs and the feed ratio.

\section{Results and discussion}

\subsection{Preparation and characterization of IL@PANI nanocomposites}

To improve the capacitance performance and mediate the morphology of PANI, organic acids have been testified to be efficient dopants (31). In the other side, ILs have been widely employed as the electrolyte of supercapacitor in view of their unique virtues. Therefore, imidazolium ILs containing carboxyl group are promising to both regulate the morphology of PANI and provide static electricity in PANI bulk, as well as high capacitance performance. However, there still are no reports on the influence of alkyl structure of ILs on the morphology of PANI. Therefore, carboxyl group contained ILs with different alkyls were designed and prepared according to the reported methods $(42,43) .{ }^{1} \mathrm{H}$ and ${ }^{13} \mathrm{C}$ NMR spectra (see the supporting information) demonstrated that carboxyl contained ILs with vinyl, methyl, butyl and hexyl groups have been successfully synthesized. Then, these ILs were used to dope PANI by in situ oxidative polymerization (as shown Scheme 1). The morphology of as-prepared IL@PANI was measured using scanning electron microscopy (SEM). As control study, PANI doped by hydrochloric acid $(\mathrm{HCl})$ and $\mathrm{CAA}$ were also prepared under the identical conditions. As shown in Figure 1, most of PANI particles doped by $\mathrm{HCl}$ presented petal-like morphology. However, when carboxyl contained ILs were used as the dopants, PANI with the morphology of maizelike nanorods were obtained. In addition, the alkyl chain length of ILs has an important effect on the morphology of PANI particles. Vinyl IL provided PANI with uniform size and maize-like nanorods, while the number of maize-like nanorods decreased with the increase of alkyl chain length in ILs. It is probably because alkyl chain length plays the key role in the physicochemical properties of IL (41). When PANI was doped by CCA, only irregular nanoparticles in the range of 200-300 nm could be prepared. The results indicated that the alkyl length of carboxyl contained ILs could direct the morphologies of PANI.

IL@PANI nanocomposites were investigated using FT-IR spectroscopy. As shown in Figure 2, PANI doped by $\mathrm{HCl}$ offered a broad absorption band at higher wavenumbers (over $2000 \mathrm{~cm}^{-1}$ ) which is representative form of conducting PANI $(45,46)$. This band was ascribed to the presence of free-charge carrier in the doped conjugate 


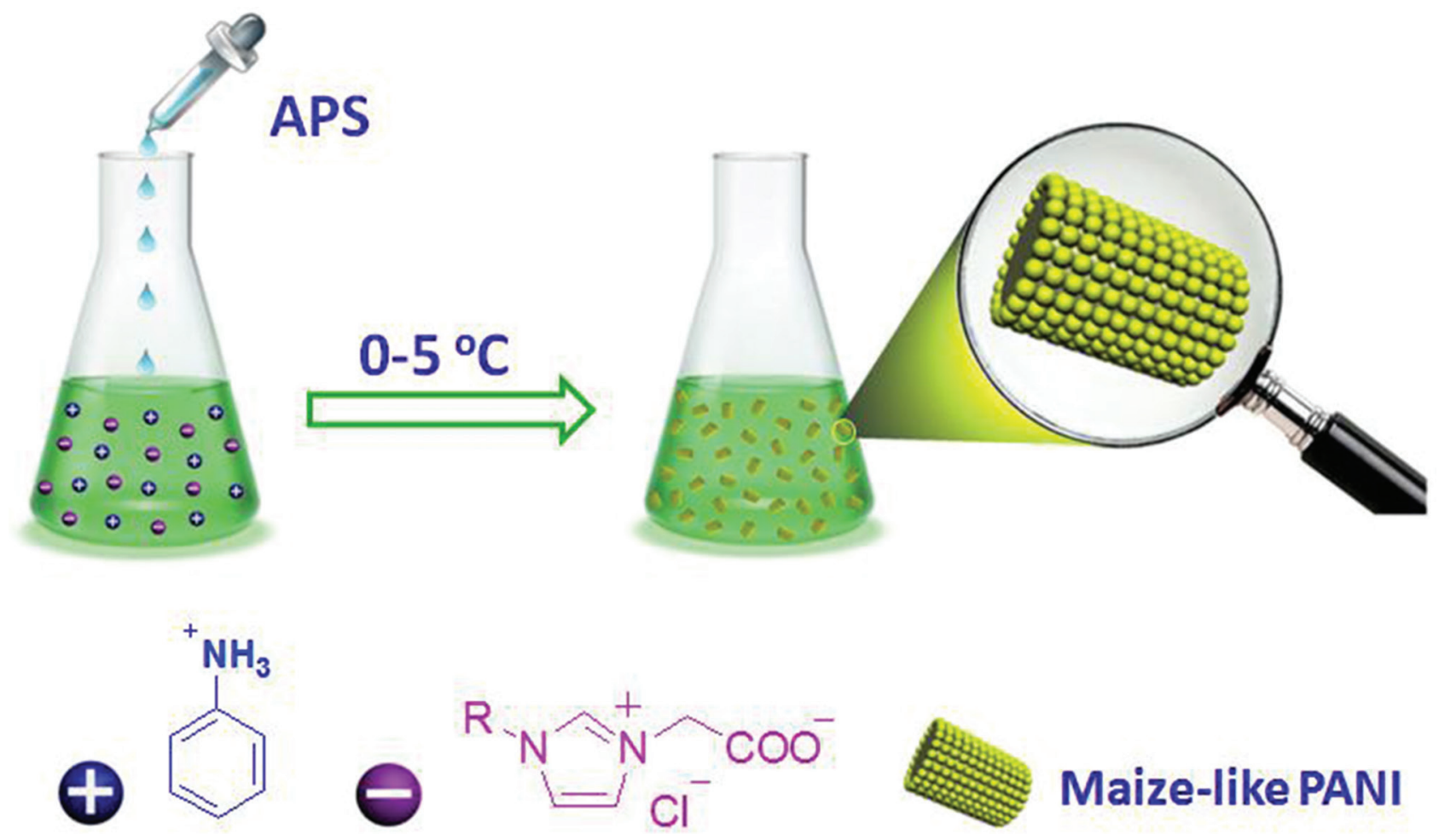

Scheme 1: Illustration of the preparation of maize-like IL@PANI nanomaterials. (R: Methyl, Vinyl, Allyl, Butyl, Hexyl).

chains. Additionally, PANI presented the distinctive absorption bands at 1571 and $1485 \mathrm{~cm}^{-1}$, which resulted from the stretching deformation of quinone and benzene rings, respectively. The absorption band at $1291 \mathrm{~cm}^{-1}$ attributed to $\pi$-electron delocalization, which was caused due to the protonation. Moreover, the characteristic band of the conducting protonated form could be observed at $1234 \mathrm{~cm}^{-1}$ and interpreted as $\mathrm{C}-\mathrm{N}^{+}$stretching vibration in the polaron structure. The absorption band at $1120 \mathrm{~cm}^{-1}$ was assigned to the vibration mode of the $-\mathrm{NH}^{+}=$ structure, which was produced during the protonation process. When PANI was doped by ILs containing different carboxylic groups, all the above typical absorption bands ascribed to PANI could be observed in the curves (Figure S10). Furthermore, the characteristic vibration bands assigned to ILs could also be distinctly observed, such as $\mathrm{C}=\mathrm{O}$ stretching vibration $\left(1741 \mathrm{~cm}^{-1}\right)$, imidazole ring vibration $\left(1568 \mathrm{~cm}^{-1}\right)$, deformation vibration of $\mathrm{C}-\mathrm{H}$ (1466 $\left.\mathrm{cm}^{-1}\right)$, stretching vibration of imidazole ring $\left(1172 \mathrm{~cm}^{-1}\right), \mathrm{C}=\mathrm{C}\left(1650 \mathrm{~cm}^{-1}\right)$ and $\mathrm{C}-\mathrm{H}\left(2800-3200 \mathrm{~cm}^{-1}\right)$ stretching vibrations. In X-ray photoelectron spectroscopy (XPS) curves (Figures 3 and S9), the characteristic peaks of $\mathrm{Cl} 2 \mathrm{p}$, O1s bands originating from [VCMIm] $\mathrm{Cl}$ and $\mathrm{N} 1 \mathrm{~s}$ band coming from PANI and [VCMIm] Cl could be clearly observed. The above results demonstrated that IL@PANI composites have been prepared successfully.

\subsection{Electrochemical properties of IL@PANI nanocomposites}

In the light of the unique maize-like morphology of IL@PANI composites, they are promising candidates for electrode materials in energy storage devices. Therefore, supercapacitors were fabricated using IL-doped PANI as the electrode material of working electrode. Then, the electrochemical performances of IL@PANI nanocomposites were evaluated using cyclic voltammetry (CV) in a three-electrode mode using $0.5 \mathrm{M} \mathrm{H}_{2} \mathrm{SO}_{4}$ aqueous solution as the electrolyte. For control investigation, electrochemical performances of $\mathrm{HCl}$ and CAA doped PANI were also measured under the identity conditions. As illustrated in Figure 4a, a couple of reversible redox peaks could be clearly perceived, indicating the typical pseudocapacitive behavior of PANI. The performance resulted from the intercalation of $\mathrm{H}^{+}$into PANI chains within the potential window of -0.2-0.8 V. Both IL@PANI and CAA@PANI composite electrodes exhibited similar $\mathrm{CV}$ profiles in the same potential window. It could be elucidated by the faradaic transition between the different oxidation states of PANI. The results manifested that IL doped PNAI could offer much higher specific capacitance than CAA or $\mathrm{HCl}$ doped PANI. To attain a comprehensive understanding 

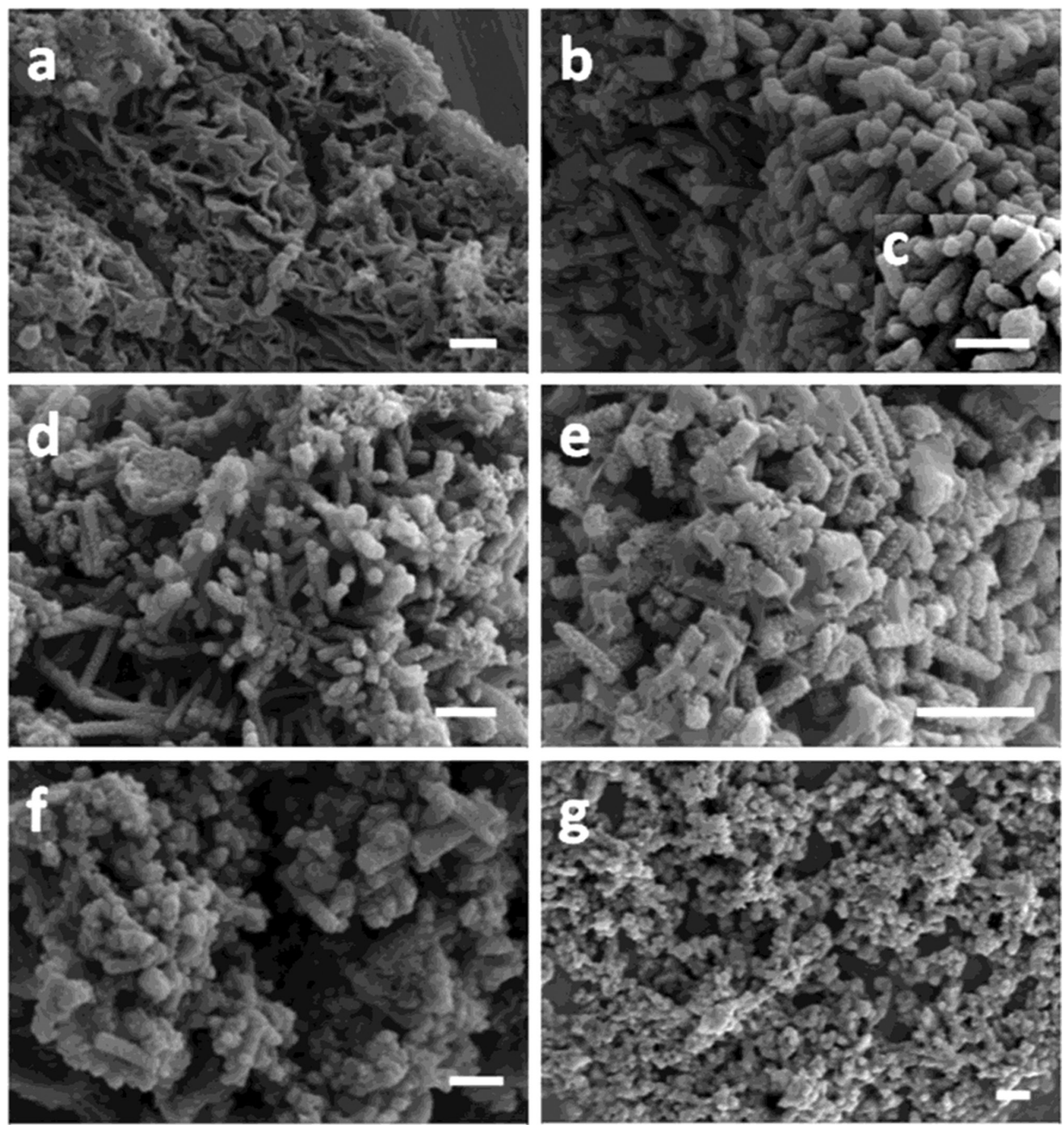

Figure 1: SEM images of PANI nanocomposites with different dopants. (a) $\mathrm{HCl}$, (b) [VCMIm]Cl, (c) magnification of b, (d) [MCMIm]Cl, (e) $[\mathrm{BCMIm}] \mathrm{Cl}$, (f) $[\mathrm{HCMIm}] \mathrm{Cl}$, (g) chloroacetic acid. All the scale bars are $1 \mu \mathrm{m}$.

of the capacitive performance of IL@PANI composites, galvanostatic charge-discharge (GCD) measurements were performed at current densities of $1 \mathrm{~A} \mathrm{~g}^{-1}$ within the potential window of $0-0.8 \mathrm{~V}$. The specific capacitance $\left(\mathrm{C}_{\mathrm{s}}\right)$ was calculated according to the following equation (47):

$$
\mathrm{C}_{\mathrm{s}}=\mathrm{C} / \mathrm{m}=(\mathrm{I} \Delta \mathrm{t}) /(\Delta \mathrm{Vm})
$$

Where $\mathrm{C}_{\mathrm{s}}$ is specific capacitance $\left(\mathrm{F} \mathrm{g} \mathrm{g}^{-1}\right)$, $\mathrm{I}$ is chargedischarge current density (A), $\Delta \mathrm{t}$ is discharging time (s), $\Delta \mathrm{V}$ is potential range during the discharge process $(\mathrm{V})$, $\mathrm{m}$ is the mass of active material within the electrode ( $\mathrm{g}$ ). Figure 4b shows the representative GCD curves of PANI composites with different dopants. Consequently, the specific capacitance of PANI with different dopants could be calculated according to the function above, and the 


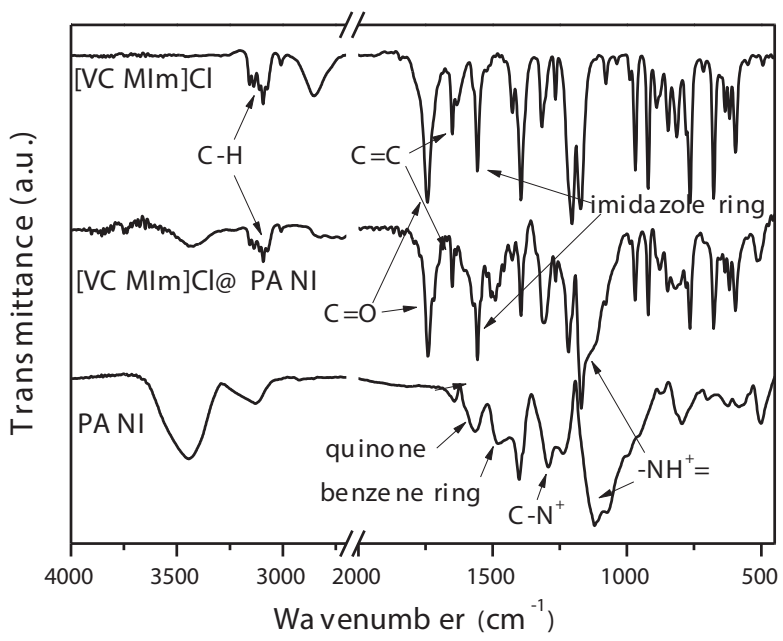

Figure 2: FTIR spectra of [VCMIm] Cl, pristine PANI and [VCMIm]Cl@ PANI nanomaterials.

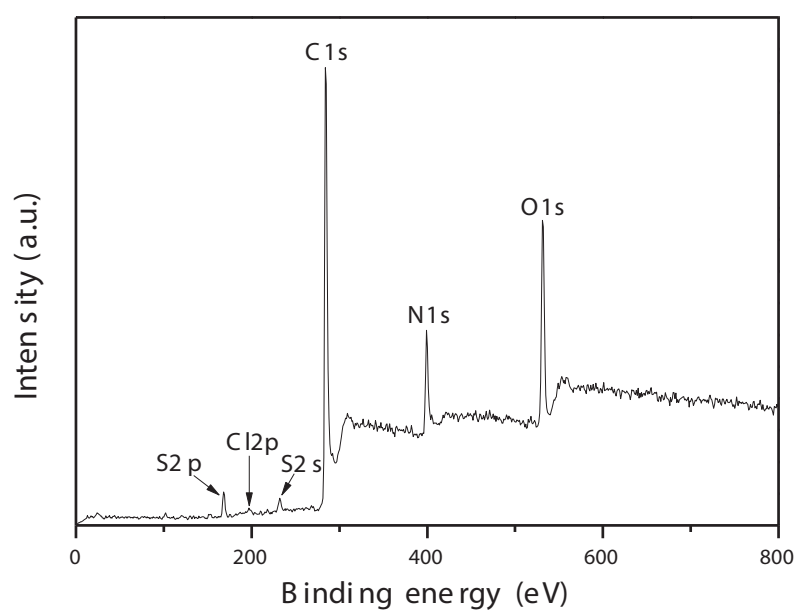

Figure 3: XPS survey scan of PANI doped by [VCMIm]Cl.

results were illustrated in Figure S11. Remarkably, the specific capacitance as high as $624 \mathrm{~F} \mathrm{~g}^{-1}$ was presented by [VCMIm]Cl doped PANI, which was much higher than those of other dopants. And the specific capacitances of the as-obtained PANI composites were in the following sequence: $[\mathrm{VCMIm}] \mathrm{Cl}\left(624 \mathrm{~F} \mathrm{~g}^{-1}\right)>[\mathrm{ACMIm}] \mathrm{Cl}\left(337 \mathrm{~F} \mathrm{~g}^{-1}\right) \approx$ [MCMIm] Cl(331 $\left.\mathrm{F} \mathrm{g}^{-1}\right)>\left[\right.$ BCMIm] Cl $\left(262 \mathrm{~F} \mathrm{~g}^{-1}\right)>[$ HCMIm] $\mathrm{Cl}\left(216 \mathrm{~F} \mathrm{~g}^{-1}\right)>\mathrm{CAA}\left(201 \mathrm{~F} \mathrm{~g}^{-1}\right)>\mathrm{HCl}\left(166 \mathrm{~F} \mathrm{~g}^{-1}\right)$. Based on the results, it could be inferred that IL-based dopants could enhanced the capacitance of PANI greatly. Additionally, some well-defined PANI composites containing ILs were summarized in Table S1. It can be found that [VMIM]Cl@PAN composite offered a higher capacitance performance than most of these well-defined PANI composites. Only when rGO was introduced, a comparable specific capacitance could be achieved. However, these low dimension carbon materials (GO, CNT) usually need high cost and complicated manipulation. Thus, IL@PANI composites offered the advantages such as easy preparation and morphology-controllability.

Normally, conductive polymers exhibit typical pseudocapacitance, and can offer higher capacitance performance, which is mainly ascribed to the surface reactions of electrode materials including electrochemical adsorption/desorption of cations and anions at the electrode/electrolyte interface and surface faradic redox reactions (4). Therefore, nitrogen-containing groups in the electrode materials can generate or increase redox reactions, electron donor capability, and electrode wettability (48). In the present study, the imidazole and carboxyl groups in ILs can improve the electrode wettability which facilitated the surface reactions of electrode materials. More importantly, ILs themselves also played the role of electrolyte in PANI bulk and could mediate the morphology of PANI. Therefore, IL-doped PANI composites could offer higher specific capacitance than those doped by $\mathrm{CAA}$ and $\mathrm{HCl}$. As far as ILs-based dopants were concerned, the specific capacitance of PANI nanocomposites decreased with the length of alkyl chain in IL. The highest performance of [VCMIm]Cl@PANI composite was probably because [VCMIm] could mediate the morphology of PANI in the polymerization process very well, and the ordered maizelike structure enhanced the utilization of PANI greatly. On the other hand, the conjugated $\pi$ electron of vinyl benefited to the interaction between [VCMIm] $\mathrm{Cl}$ and PANI. These factors synergistically induced the much higher specific capacitance of [VCMIm]Cl@PANI composite than PANI doped by other ILs.

Cyclic voltammetry curves of [VCMIm]Cl@PANI electrode material performed in $0.5 \mathrm{M} \mathrm{H}_{2} \mathrm{SO}_{4}$ at the scan rates from 5 to $50 \mathrm{mV} \mathrm{s}^{-1}$ were indicated in Figure $5 \mathrm{a}$. It could be seen that the nanocomposites exhibited similar curves as the scan rate increasing. A series of well defined quasi-reversible redox peaks could be found in the potential range from -0.2 to $0.8 \mathrm{~V}$. The supercapacitors presented the current response with the reductive peak shifting positively and the oxidative peak shifting negatively. Simultaneously, the peak-peak potential separation $\left(\Delta \mathrm{E}_{\mathrm{p}}\right)$ enhanced slowly with the increasing of the scan rate, which should be attributed to the increasing electrochemical polarization at higher scan rate (49). Figure 5b illustrated the CV curves of [VCMIm] Cl@PANI electrode materials with different compositions of [VCMIm] $\mathrm{Cl}$ and PANI. It could be seen clearly that [VCMIm]Cl@PANI composite with feed ratio of 1:1 was of the highest current density, indicating a higher specific capacitance. To gain deep insight into the electrochemical 

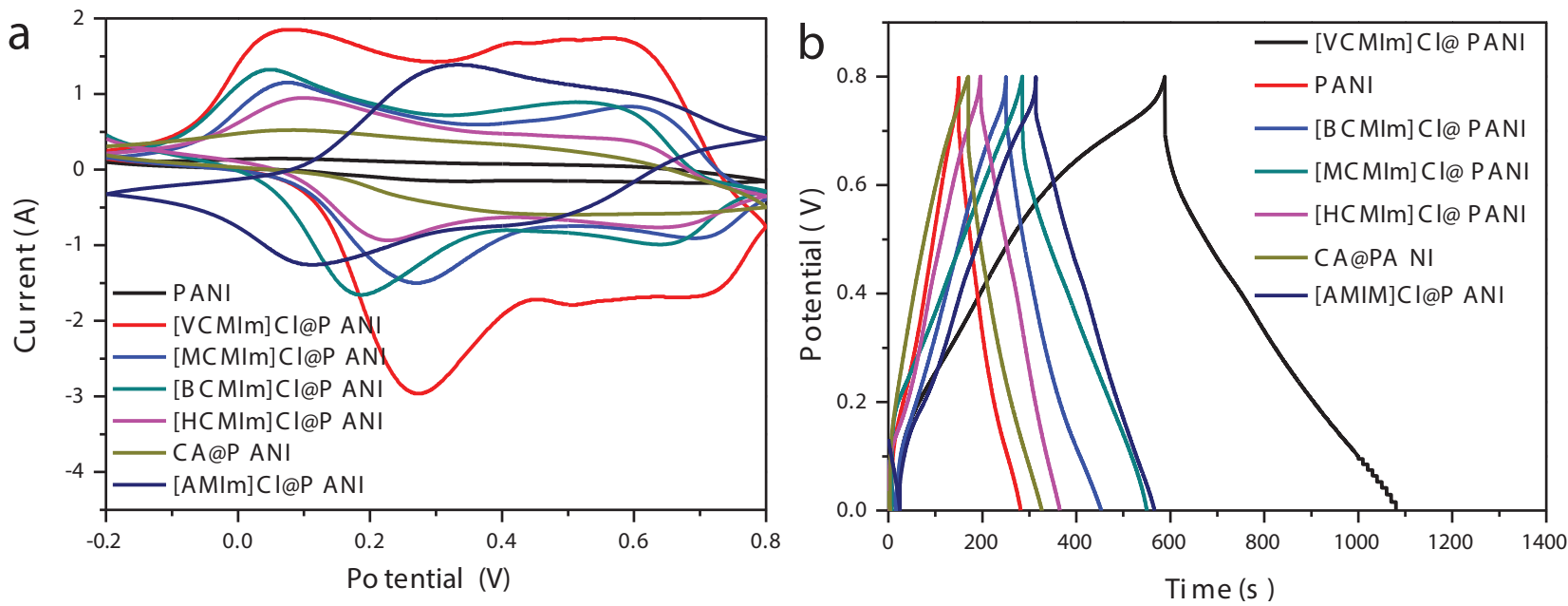

Figure 4: Electrochemical performances of IL@PANI electrode materials for supercapacitors. (a) Cyclic voltammetry profiles of PANI composites with different dopants at a scan rate of $50 \mathrm{mV} \mathrm{s}^{-1}$. (b) Galvanostatic charge/discharge curves of PANI nanocomposites with different dopants at current densities of $1.0 \mathrm{~A} \mathrm{~g}^{-1}$.
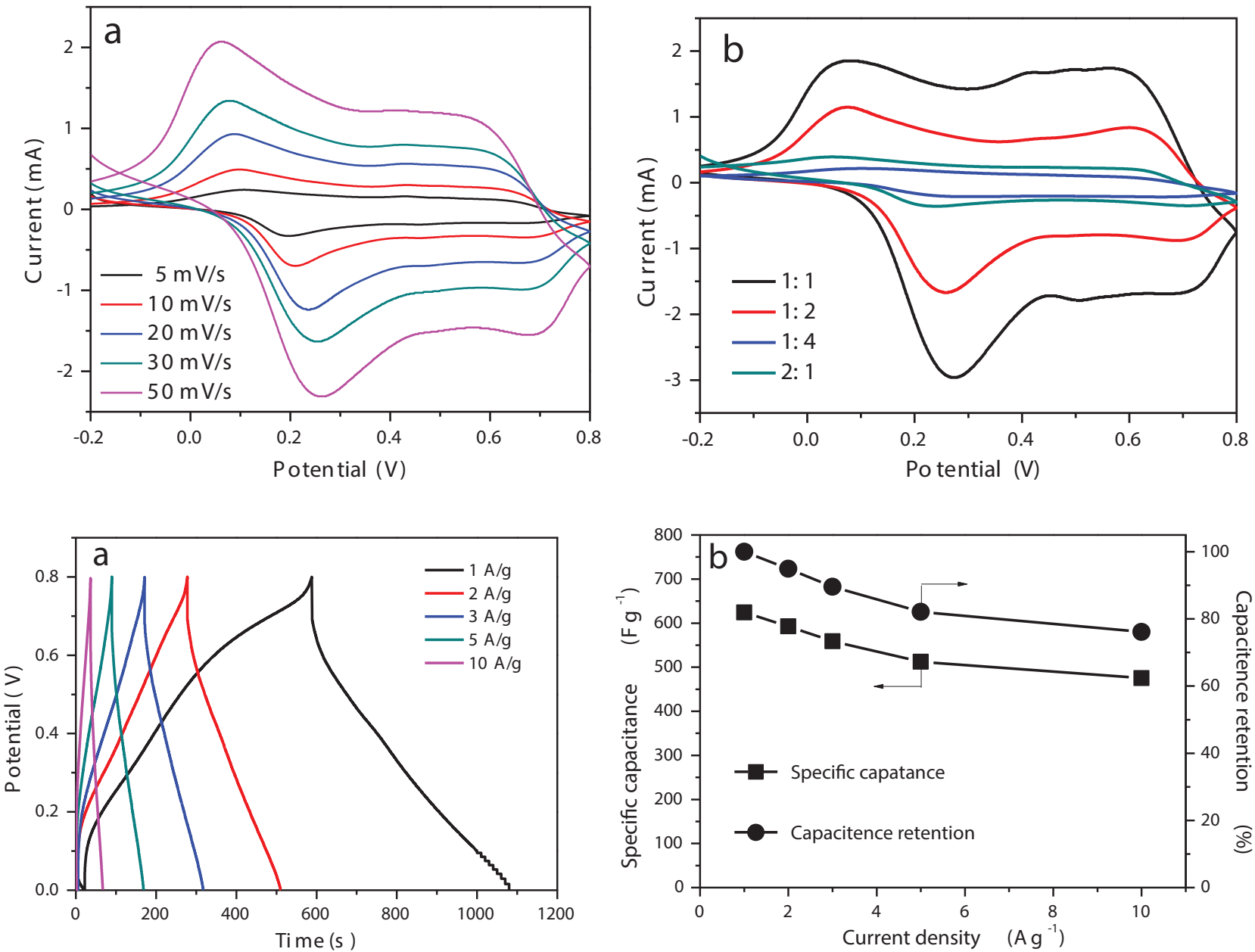

Figure 5: Electrochemical performances of [VCMIm]Cl@PANI nanocomposite for supercapacitor. (a) CV curves within the potential window from -0.2 to $0.8 \mathrm{~V}$ at different scan rates; (b) $\mathrm{CV}$ curves with the potential window from -0.2 to $0.8 \mathrm{~V}$ with different [VCMIm] Cl/[An] molar ratios at a scan rate of $50 \mathrm{mV} \mathrm{s}^{-1}$ in $0.5 \mathrm{M} \mathrm{H}_{2} \mathrm{SO}_{4}$; (c) Galvanostatic charge/discharge curves of [VCMIm]Cl@PANI electrode at various current densities; (d) Specific capacitances and capacitance retention at various current densities. 
capacitive performances of [VCMIm]Cl@PANI composites, GCD measurements were conducted at different current densities of 1-10 $\mathrm{A} \mathrm{g} \mathrm{g}^{-1}$, and the results were summarized in Figure 5c. As it showed, all the curves maintained almost the triangle shape in the potential range from 0 to $0.8 \mathrm{~V}$, which indicated its sustainable behavior in the broad current range. According to these charge/discharge curves at the different current densities, the variations of the specific capacitance of [VCMIm]Cl@PANI composites with the current density were plotted together in Figure $5 \mathrm{~d}$ for comparison. Obviously, the specific capacitance decreased with the increase of the current density. The specific capacitance decreased from 624 to $592.5 \mathrm{~F} \mathrm{~g}^{-1}$ as the current density increasing from 1 to $2 \mathrm{~A} \mathrm{~g}^{-1}$, and $95.0 \%$ capacitance could be retained. When the current density was as high as $10 \mathrm{~A} \mathrm{~g}^{-1}, 76 \%$ specific capacitance could still be maintained $\left(475 \mathrm{~F} \mathrm{~g} \mathrm{~g}^{-1}\right)$. These results attested that the [VCMIm]Cl@PANI composite electrode had excellent sustainability at high current and good rate capability.

Electrochemical impedance spectrum is a powerful technique to testify the virtues of the electrode materials during the redox process. As depicted in Figure S12, it could be found that Nyquist plots of IL@PANI composites were composed of an arc in the high frequency region and a nearly straight line in the low frequency region. The nearly vertical arm of the AC impedance in the low frequency region indicated a good capacitive behavior, representative of fast ion diffusion and adsorption in/on the electrode material (50). The charge transfer resistance value could be acquired from the span of the single semicircle along the $\mathrm{x}$-axis from high to low frequency. Thus, there were lower charge transfer resistances in IL@PANI composites. Their lower charge transfer resistances were possibly due to the synergistic effect of redox processes of PANI and the presence of ILs (51).
The corresponding Ragone plot (power density versus energy density) of [VCMIm]Cl@PANI with molar ratio of 1:1 was illustrated in Figure 6a. The energy density (E) and power density (P) were calculated according to the following equations:

$$
\begin{aligned}
& E=(1 / 2) C V^{2} \\
& P=E / \Delta t
\end{aligned}
$$

Where $\mathrm{C}$ is the capacitance of materials, $\mathrm{V}$ is the voltage decrease, $\mathrm{E}$ is the energy density and $\Delta t$ is the time spent in discharge.

As indicated in Figure 6a, the power energy density of the materials increased rapidly as the energy density decreases. And the energy density could achieve $55.5 \mathrm{Wh}$ $\mathrm{kg}^{-1}$ at a power density of $400.4 \mathrm{~W} \mathrm{~kg}^{-1}$ when the [VCMIm] $\mathrm{Cl} /[\mathrm{An}]$ molar ratio was 1:1. Moreover, the energy density still remained $42.2 \mathrm{Wh} \mathrm{kg}^{-1}$ even at a power density as high as $3997.9 \mathrm{~W} \mathrm{~kg}^{-1}$. The results attested that [VCMIm] Cl@PANI composite was of remarkable electrochemical properties with high energy density and power output.

For electrode materials of supercapacitor, the cycling performance also plays a very important role. Thus, the specific capacitance of [VCMIm]Cl@PANI electrode material as a function of cycle number based on charge/ discharge curves at the current density of $1.0 \mathrm{~A} \mathrm{~g}^{-1}$ was summarized in Figure $6 \mathrm{~b}$. It could be found that almost half capacitance of pristine PANI lost after 200 cycles, indicating very short cycle life of pristine PANI. Compared with the pristine PANI, only a little decrease in the specific capacitance of [VCMIm]Cl@PANI electrode material was produced before 400 cycles, and $94.6 \%$ of the initial value could be remained. Thus, the introduction of [VCMIm]Cl could improve the cycle life of PANI greatly. After that, the
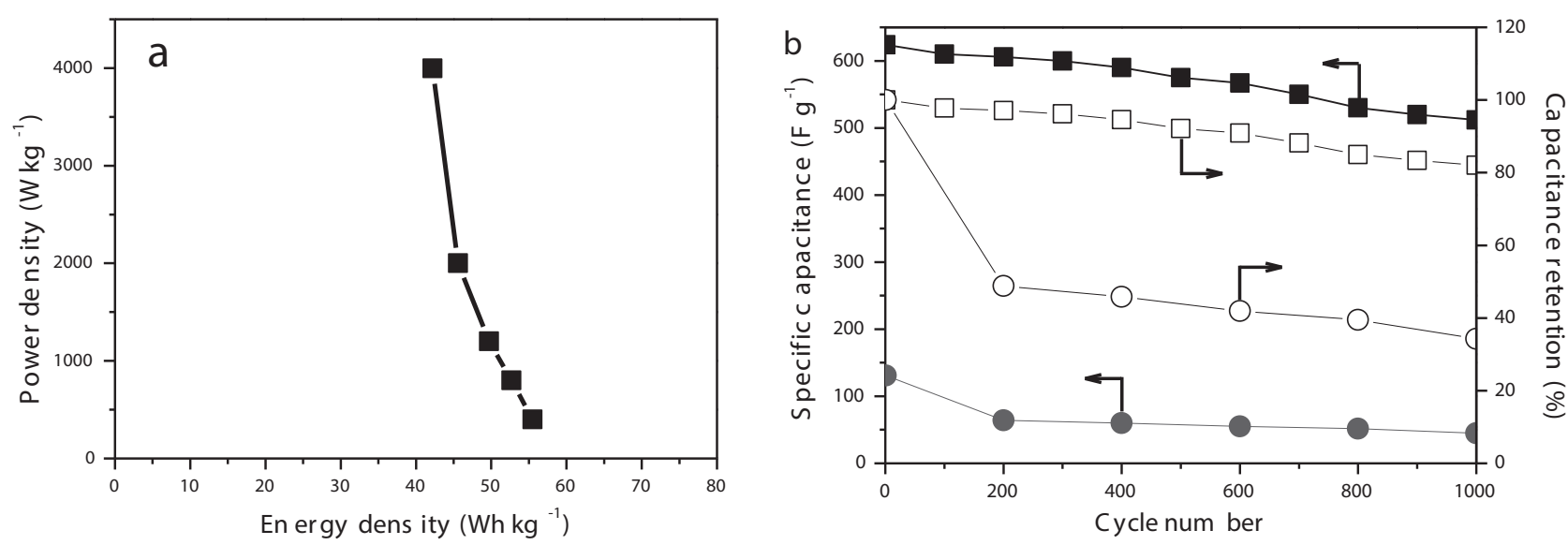

Figure 6: (a) Ragone plots (power density vs energy density) of [VCMIm]Cl@PANI electrodes. (b) Variations of the specific capacitance and capacitance retention of PANI electrodes as a function of cycle number. (square: [VCMIm]Cl@PANI; circle: pristine PANI) 
specific capacitance decreased quickly, and only $82 \%$ of the initial specific capacitance could be maintained after 1000 cycles. The fast decrease in specific capacitance of [VCMIm]Cl@PANI electrode materials was probably ascribed to the swelling and shrinkage of conducting PANI during the long time charge/discharge processes $(52,53)$. Fortunately, a recent report demonstrated that superior stability of conductive polymer supercapacitors could be achieved through combining electrochemically active polymers with redox-active electrolytes (25).

\section{Conclusions}

In summary, we developed a scalable solution-based approach to PANI nanocomposite with controllable morphology, which could be achieved through chemical oxidation polymerization in the presence of ILs containing carboxyl group. SEM images indicated that maize-like nanorods could be obtained, and the alkyl length of ILs played an important role on the morphology of IL@PANI composites. The capacitance performance of IL@PANI composites were thoroughly investigated by cyclic voltammetry, galvanostatic charge/discharge, electrochemical impedance spectroscopy and cycle life measurements. The results revealed that the highest specific capacitance (624 $\mathrm{F} \mathrm{g}^{-1}$ at the current density of $1 \mathrm{~A} \mathrm{~g} \mathrm{~g}^{-1}$ ) could be attended when PANI was doped by [VCMIm] Cl with the feed ratio of 1:1. Additionally, $82 \%$ capacitance could be retained after 1000 cycles. Therefore, our strategy renders a significant step forward to bringing IL@PANI electrode material to versatile applications in supercapacitor and other electronic systems.

Acknowledgements: The authors are grateful for the financial support given by the National Natural Science Foundation of China (Grant No. 21774101, 21474080).

\section{References}

1. Arico A.S., Bruce P., Scrosati B., Tarascon J.M., Schalkwijk W., Nanostructured materials for advanced energy conversion and storage devices. Nat Mater, 2005, 4, 366-377.

2. Miller J.R., Simon P., Electrochemical capacitors for energy management. Science, 2008, 321, 651-652.

3. Kotz R., Carlen M., R\&D considerations for the performance and application of electrochemical capacitors. Electrochim Acta, 2007, 53, 1083-1091.

4. Simon P., Gogotsi Y., Materials for electrochemical capacitors. Nat Mater, 2008, 7, 845-854.
5. Yuan C.Z., Gao B., Shen L.F., Yang S.D., Hao L., Lu X.J., et al., Hierarchically structured carbon-based composites: Design, synthesis and their application in electrochemical capacitors. Nanoscale, 2011, 3, 529-545.

6. Zhang L.L., Zhao X.S., Carbon-based materials as supercapacitor electrodes. Chem Soc Rev, 2009, 38, 2520-2531.

7. Zhang H., Cao G.P., Yang Y.S., Carbon nanotube arrays and their composites for electrochemical capacitors and lithium-ion batteries. Energ Environ Sci, 2009, 2, 932-943.

8. Wang G., Zhang L., Zhang J., A review of electrode materials for electrochemical supercapacitors. Chem Soc Rev, 2012, 41, 797-828.

9. Pech D., Brunet M., Durou H., Huang P.H., Mochalin V., Gogotsi Y., et al., Ultrahigh-power micrometre-sized supercapacitors based on onion-like carbon. Nat Nanotech, 2010, 5, 651-654.

10. Wang D.W., Li F., Liu M., Lu G.Q., Cheng H.M., 3D aperiodic hierarchical porous graphitic carbon material for high-rate electrochemical capacitive energy storage. Angew. Chem Int Ed, 2008, 47, 373-376.

11. Liu C.G., Yu Z.N., Neff D., Zhamu A., Jang B.Z., Graphene-based supercapacitor with an ultrahigh energy density. Nano Lett, 2010, 10, 4863-4868.

12. Zhu Y.W., Murali S., Stoller M.D., Ganesh K.J., Cai W.W., Ferreira P.J., et al., Carbon-based supercapacitors produced by activation of graphene. Science, 2011, 332, 1537-1541.

13. Jiang H., Lee P.S., Li C.Z., 3D carbon based nanostructures for advanced supercapacitors. Energy Environ Sci, 2013, 6, 41-53.

14. Bose S., Kuila T., Mishra A.K., Rajasekar R., Kimc N.H., Lee J.H., Carbon-based nanostructured materials and their composites as supercapacitor electrodes. J Mater Chem, 2012, 22, 767-784.

15. Wei W.F., Cui X.W., Chen W.X., Ivey D.G., In situ polymerization and reduction to fabricate gold nanoparticle-incorporated polyaniline as supercapacitor electrode materials. Chem Soc Rev, 2011, 40, 1697-1721.

16. Deng W.T., Ji X.B., Chen Q.Y., Banks C.E., Electrochemical capacitors utilising transition metal oxides: an update of recent developments. RSC Adv, 2011, 1, 1171-1178.

17. Wei T.Y., Chen C.H., Chien H.C., Lu S.Y., Hu C.C., A cost-effective supercapacitor material of ultrahigh specific capacitances: Spinel nickel cobaltite aerogels from an epoxide-driven sol-gel process. Adv Mater, 2010, 22, 347-351.

18. Snook G.A., Kao P., Best A.S., Conducting-polymer-based supercapacitor devices and electrodes. J Power Sources, 2011, 196, 1-12.

19. Zhao Y., Liu B.R., Pan L.J., Yu G.H., 3D nanostructured conductive polymer hydrogels for high-performance electrochemical devices. Energy Environ Sci, 2013, 6, 2856-2870.

20. Richard Prabhu Gnanakan S., Murugananthem N., Subramania A., Organic acid doped polythiophene nanoparticles as electrode material for redox supercapacitors. Polym Adv Technol, 2011, 22, 788-793.

21. Ran F., Tan Y.T., Dong W.J., Liu Z., Kong L.B., Kang L., In situ polymerization and reduction to fabricate gold nanoparticleincorporated polyaniline as supercapacitor electrode materials. Polym Adv Technol, 2018, 29, 1697-1705.

22. Bhadra S., Khastgir D., Singha N.K., Lee J.H., Progress in preparation, processing and applications of polyaniline. Prog Polym Sci, 2009, 34, 783-810. 
23. W. Xing, X. Yuan, S.P. Zhuo, C.C. Huang, Preparation of polyaniline-coated mesoporous carbon and its enhanced electrochemical properties. Polym Adv Technol, 2009, 20, 1179-1182.

24. Bolagam R., Boddula R., Srinivasan P., Synthesis of highly crystalline polyaniline with the use of (Cyclohexylamino)1-propanesulfonic acid for supercapacitor. J Appl Electrochem, 2015, 45, 51-56.

25. Singu B.S., Palaniappan S., Yoon K.R., Polyaniline-nickel oxide nanocomposites for supercapacitor. J Appl Electrochem, 2016, 46, 1039-1047.

26. Zhou H.H., Chen H., Luo S.L., Lu G.W., Wei W.Z., Kuang Y.F., The effect of the polyaniline morphology on the performance of polyaniline supercapacitors. J Solid State Electrochem, 2005, 9, 574-580.

27. Ran F., Tan Y.T., Liu J., Zhao L., Kong L.B., Luo Y.C., et al., Preparation of hierarchical polyaniline nanotubes based on self-assembly and its electrochemical capacitance. Polym Adv Technol, 2012, 23, 1297-1301.

28. Xia C., Xie Y., Wang Y., Wang W., Du H., Tian F., Preparation and capacitance performance of polyaniline/titanium nitride nanotube hybrid. J Appl Electrochem, 2013, 43, 1225-1233.

29. Kulkarni M.V., Viswanath A.K., Marimuthu R., Seth T., Synthesis and characterization of polyaniline doped with organic acids. J Polym Sci Part A Polym Chem, 2004, 42, 2043-2049.

30. Yuan J., Mecerreyes D., Poly(ionic liquid)s: An update. Antonietti M., Prog Polym Sci, 2013, 38, 1009-1036.

31. Lu J., Yan F., Texter J., Advanced applications of ionic liquids in polymer science. Prog Polym Sci, 2009, 34, 431-448.

32. Parvulescu V.I., Hardacre C., Catalysis in ionic liquids. Chem Rev, 2007, 107, 2615-2665.

33. Armand M., Endres F., MacFarlane D.R., Ohno H., Scrosati B., Ionic-liquid materials for the electrochemical challenges of the future. Nat Mater, 2009, 8, 621-629.

34. Eshetu G.G., Armand M., Scrosati B., Passerini S., Energy Storage Materials Synthesized from Ionic Liquids. Angew Chem Int Ed, 2014, 53, 13342-13359.

35. Macfarlane D.R., Tachikawa N., Forsyth M., Pringle J.M., Howlett P.C., Elliott G.D., et al., Energy applications of ionic liquids. Energy Environ Sci, 2014, 7, 232-250.

36. Zhang W., Yuan C., Guo J., Qiu L., Yan F., Supramolecular ionic liquid gels for quasi-solid-state dye-sensitized solar cells. ACS Appl Mater Interfaces, 2014, 6, 8723-8728.

37. Pandey G.P., Rastogi A.C., Westgate C.R., All-solid-state supercapacitors with poly(3,4-ethylenedioxythiophene)-coated carbon fiber paper electrodes and ionic liquid gel polymer electrolyte. J Power Sources, 2014, 245, 857-865.

38. Attri P., Park D.H., Lee S.H., Kim Y.S., Kim Y.B., Kwon G.C., et al., Physicochemical Properties of Polyaniline-Ionic Liquid Mixtures and Their Application in Dye-Sensitized Solar Cells. Sci Adv Mater, 2015, 7(12), 2583-2595.

39. Pahovnik D., Zagar E., Kogej K., Vohlidal J., Zigon M., Polyaniline nanostructures prepared in acidic aqueous solutions of ionic liquids acting as soft templates. Eur Polym J, 2013, 49, 1381-1390.

40. Tokuda H., Hayamizu K., Ishii K., Susan Md A.B.H., Watanabe M., Physicochemical properties and structures of room temperature ionic liquids. 2. variation of alkyl chain length in imidazolium cation. J Phys Chem B, 2005, 109, 6103-6110.

41. Kim T.Y., Lee H.W., Stoller M., Dreyer D.R., Bielawski C.W., Ruoff R.S., Suh K.S., High-performance supercapacitors based on poly(ionic liquid)-modified graphene electrodes. ACS Nano, 2011, 5, 436-442.

42. Xiong Y.B., Wang H., Wang R.M., Wang Z., Novel imidazoliumbased poly(ionic liquid)s: preparation, characterization, and absorption of $\mathrm{CO}_{2}$. Polym Adv Tech, 2012, 23, 835-840.

43. Wang Z., Xiong Y.B., Wang H., Wang R.M., Wang Y.P., Preparation and conductive behavior of polyaniline/ionicliquid/montimorillonite nanocomposites. Polym Acta Sin, 2010, 812-818.

44. Wei Z.X., Zhang Z.M., Wan M.X., Formation mechanism of self-assembled polyaniline micro/nanotubes. Langmuir, 2002, 18, 917-921.

45. Ping Z., In situ FTIR-attenuated total reflection spectroscopic investigations on the base-acid transitions of polyaniline. Base-acid transition in the emeraldine form of polyaniline. J Chem Soc Faraday Trans, 1996, 92, 3063-3067.

46. Sedenkova I., Trchova M., Stejskal J., Thermal degradation of polyaniline films prepared in solutions of strong and weak acids and in water-FTIR and Raman spectroscopic studies. Polym Degrad Stab, 2008, 93, 2147-2157.

47. Zhang J., Zhao X.S., Conducting polymers directly coated on reduced graphene oxide sheets as high-performance supercapacitor electrodes. J Phys Chem C, 2012, 116, 5420-5426.

48. Wang Y.G., Song Y.F., Xia Y.Y., Electrochemical capacitors: mechanism, materials, systems, characterization and applications. Chem Soc Rev, 2016, 45, 5925-5950.

49. Gennaro A., Isse A.A., Severin M.G., Vianello E., Bhugun I., Saveant J.M., Mechanism of the electrochemical reduction of carbon dioxide at inert electrodes in media of low proton availability. J Chem Soc Faraday Trans, 1996, 92, 3963-3968.

50. Li Z.F., Zhang H.Y., Liu Q., Liu Y.D., Stanciu L., Xie J., Covalentlygrafted polyaniline on graphene oxide sheets for high performance electrochemical supercapacitors. Carbon, 2014, 71, 257-267.

51. Xie H., Zhu Y., Wu Y., Wu Z., Liu E., The effect of hydroquinone as an electrolyte additive on electrochemical performance of the polyaniline supercapacitor. Mater Res Bull, 2014, 50, 303-306.

52. Li J., Xie H.Q., Li Y., Liu J., Lim Z.X. Electrochemical properties of graphene nanosheets/polyaniline nanofibers composites as electrode for supercapacitors. J Power Sources, 2011, 196, 10775-10781.

53. Wang H.L., Hao Q.L., Yang X.J., Lu L.D., Wang X.A., A nanostructured graphene/polyaniline hybrid material for supercapacitors. Nanoscale, 2010, 2, 2164-2170. 